\title{
A counseling framework for moderate-penetrance colorectal cancer susceptibility genes
}

\author{
Bryson W. Katona, MD PhD ${ }^{1}$, Matthew B. Yurgelun, MD², Judy E. Garber, MD MPH², \\ Kenneth Offit, MD MPH ${ }^{3,4}$, Susan M. Domchek, MD ${ }^{1}$, Mark E. Robson, $\mathrm{MD}^{3,4}$ and \\ Zsofia K. Stadler, MD $\mathrm{MD}^{3,4}$
}

The inclusion of moderate-penetrance cancer susceptibility genes in multigene panel testing poses challenges regarding the optimal management of individuals found to have pathogenic variants in these genes. A recently published counseling framework has provided evidence-based guidance for moderate-penetrance breast and ovarian cancer genes. ${ }^{1}$ However, no such framework exists for moderate-penetrance colorectal cancer (CRC) susceptibility genes, including CHEK2, APC ${ }^{\star} 11307 \mathrm{~K}$, and monoallelic MUTYH, which are among the most common multigene panel testing findings. ${ }^{2}$ Currently the only recommendations to address this risk are from the National Comprehensive Cancer Network, which recommends that individuals who carry pathogenic CHEK2 or $A P C^{\star} \mathrm{I} 1307 \mathrm{~K}$ variants irrespective of family history, or monoallelic MUTYH with a family history of CRC, undergo earlier and more frequent CRC screening, similar to individuals with a first-degree relative with CRC. ${ }^{3}$ Whether early and increased CRC screening is truly justified for such carriers in the absence of a family history of CRC is unknown.

To better elucidate the appropriate timing of colonoscopy initiation, we calculated the cumulative lifetime risk (CLTR) of CRC as a multiple of the US Surveillance, Epidemiology, and End Results Program (SEER) estimates of ever developing $\mathrm{CRC}$ and the observed risk for selected genetic variants (CHEK2 1100delC, CHEK2 I157K, APC ${ }^{\star} I 1307 \mathrm{~K}$, monoallelic $M U T Y H)$. Population age-specific incidence rates for CRCs were obtained from the 2010-2014 SEER cancer statistics for all races. ${ }^{4}$ Average relative-risk multipliers were derived from a systematic meta-analysis. ${ }^{5}$ We estimated 5 -year and CLTR using previously described methods and applied the estimated odds ratio (OR) for each genetic variant to population agespecific incidence data. ${ }^{6}$

\section{MODERATE-PENETRANCE CRC GENES CHEK2}

Pathogenic CHEK2 variants are present in $~ 1 \%$ of Caucasians of European descent, ${ }^{7}$ with evidence for CRC risk limited to the two most common founder mutations 1100delC and
I157T. Although multiple reports demonstrated a nonsignificant CRC risk, ${ }^{8}$ two meta-analyses reported a modestly increased CRC risk with ORs of 1.88 (95\% confidence interval (CI), $1.29-2.73)^{5}$ and 2.11 (95\% CI, $\left.1.41-3.16\right)^{9}$ in CHEK2 1100 delC carriers. However, given some irregularities in the analysis, the reported $2.11 \mathrm{OR}^{9}$ appears to have been an overestimation with adjusted data suggesting an OR of 1.8 $(95 \% \mathrm{CI}, 1.2-2.7),{ }^{10}$ similar to that in the report by Ma et al. ${ }^{5}$ In comparison, the relative risk of CRC in individuals with a family history of CRC is reported as 2.25 (95\% CI, 2.0-2.53). ${ }^{11}$ For CHEK2 I157T, a meta-analysis demonstrated an OR of 1.61 (95\% CI, 1.40-1.87) for CRC, ${ }^{12}$ similar to that in the meta-analysis by Ma et al. ${ }^{5}$ (OR 1.56; 95\% CI, 1.32-1.84). CRC risk may be increased in CHEK2 carriers with a CRC family history, thereby implicating family history as a potentially important risk modifier. ${ }^{12}$ However, there is no strong evidence that CHEK2 is associated with CRC before age 50 , nor an earlier age of CRC diagnosis. ${ }^{8}$

\section{$A P C * \mid 1307 \mathrm{~K}$}

The $A P C^{\star} \mathrm{I} 1307 \mathrm{~K}$ founder germ line variant, present in $7 \%$ of Ashkenazi Jews, is associated with a 1.96 (95\% CI, 1.37-2.79) OR for CRC risk in Ashkenazi Jews., ${ }^{5,13}$ Age at CRC diagnosis in $A P C^{*} \mathrm{I} 1307 \mathrm{~K}$ carriers is not different from that in noncarriers. ${ }^{13}$ Association of $A P C^{\star} 11307 \mathrm{~K}$ with a family history is less clear with studies among CRC patients reporting a family history of $\mathrm{CRC}$ ranging from 0 to $28 \%{ }^{13}$

\section{Monoallelic MUTYH}

Monoallelic MUTYH mutations are among the more common germ line abnormalities discovered via multigene panel testing, regardless of the population being tested, with a $\sim 2 \%$ prevalence. $^{2}$ CRC risk estimates associated with monoallelic MUTYH mutations are conflicting. The Colon Cancer Family Registry examined 223 monoallelic MUTYH carriers and their relatives estimating that such individuals had a cumulative $0.8 \%$ risk of CRC through age 50 , regardless of CRC family history, versus $0.3 \%$ risk for noncarriers. ${ }^{14}$

${ }^{1}$ University of Pennsylvania Perelman School of Medicine, Philadelphia, Pennsylvania, USA; ${ }^{2}$ Dana-Farber Cancer Institute, Harvard Medical School, and Brigham \& Women's Hospital, Boston, Massachusetts, USA; ${ }^{3}$ Clinical Genetics Service, Department of Medicine, Memorial Sloan-Kettering Cancer Center, New York, New York, USA; ${ }^{4}$ Department of Medicine, Weill Cornell Medical College, New York, New York, USA. Correspondence: Zsofia K. Stadler (stadlerz@mskcc.org)

Submitted 4 December 2017; accepted 17 January 2018; advance online publication 1 March 2018. doi:10.1038/gim.2018.12 
However, a large meta-analysis including $>25,000$ MUTYH monoallelic carriers and $>18,000$ controls reported only a very slight increase in CRC risk (OR 1.17; 95\% CI, 1.01-1.34) that was based on weak overall evidence. ${ }^{5}$

\section{MANAGING CRC RISK}

Given uncertainties in the management of individuals with moderate-penetrance CRC gene variants, we estimated CRC risk associated with these variants to help better define possible CRC risk-reduction strategies. Estimated 5-year and CLTR of CRC for average-risk individuals and moderatepenetrance gene mutation carriers are shown in Table 1. For the average-risk individual, where current guidelines recommend initiating screening colonoscopy at age 50, a CLTR of CRC of $0.6 \%$ is reached by age $50-54 .{ }^{3}$ However, for CHEK2 1100delC, CHEK2 I157T, and $A P C^{\star}{ }^{\top} 11307 \mathrm{~K}$ carriers, this same level of CLTR of CRC is reached by age 45-49. Therefore, if initiation of CRC screening is based on the average-risk individual's CLTR of CRC, earlier initiation of screening colonoscopy in this timeframe in pathogenic CHEK2 and $A P C^{\star} \mathrm{I} 1307 \mathrm{~K}$ carriers without a family history of CRC would seem reasonable (Table 2). Although our data is based on risk associated with CHEK2 1100delC and CHEK2 I157T, we would recommend considering all pathogenic CHEK2 mutation carriers equivalently given the current paucity of data on CRC risk associated with other CHEK2 variants. For monoallelic MUTYH carriers, cumulative risk of CRC is $0.7 \%$ by age $50-54$, similar to individuals at average risk. Therefore, using similar reasoning, there does not appear to be an indication for earlier initiation of colonoscopy in monoallelic MUTYH carriers in the absence of a family history, consistent with current National Comprehensive Cancer Network recommendations. $^{3}$

While such recommendations apply to patients with no family history of CRC, the presence of a family history increases risk, as individuals with a first-degree relative with

Table 2 Colonoscopy screening recommendations for individuals with moderate-penetrance mutations in selected genes

\begin{tabular}{|c|c|c|c|}
\hline Gene & $\begin{array}{l}\text { Family history } \\
\text { of CRC }\end{array}$ & $\begin{array}{l}\text { Age to start } \\
\text { CRC screening }\end{array}$ & $\begin{array}{l}\text { Screening } \\
\text { interval if } \\
\text { no adenomas }\end{array}$ \\
\hline \multirow[t]{3}{*}{ No mutation ${ }^{a}$} & No & 50 & 10 years \\
\hline & Yes ( $\geq 1$ FDR) & $40^{b}$ & $5-10$ years \\
\hline & $\begin{array}{l}\text { Yes }(\geq 1 \text { SDR } \\
<50 \text { yo })\end{array}$ & 50 & $5-10$ years \\
\hline \multirow[t]{2}{*}{$A P C \star \mid 1307 K$} & No & 45 & 5 years \\
\hline & Yes & 45 or per $\mathrm{FHx}$ & 5 years \\
\hline \multirow[t]{2}{*}{ CHEK2 } & No & 45 & 5 years \\
\hline & Yes & 45 or per FHx & 5 years \\
\hline \multirow{2}{*}{$\begin{array}{l}\text { Monoallelic } \\
\text { MUTYH }\end{array}$} & No & 50 & 10 years \\
\hline & Yes & 50 or per $\mathrm{FHx}$ & 5 years \\
\hline
\end{tabular}

CRC, colorectal cancer; FDR, first-degree relative; FHx, family history; SDR, seconddegree relative.

${ }^{a}$ Recommendations based on guidelines from the National Comprehensive Cancer Network (ref. 3). ${ }^{\mathrm{b}} 40$ years old or 10 years earlier than the age of the youngestonset CRC.

Table 1 Estimated 5-year and lifetime colon and rectal cancer risk for individuals with moderate-penetrance mutations in selected genes

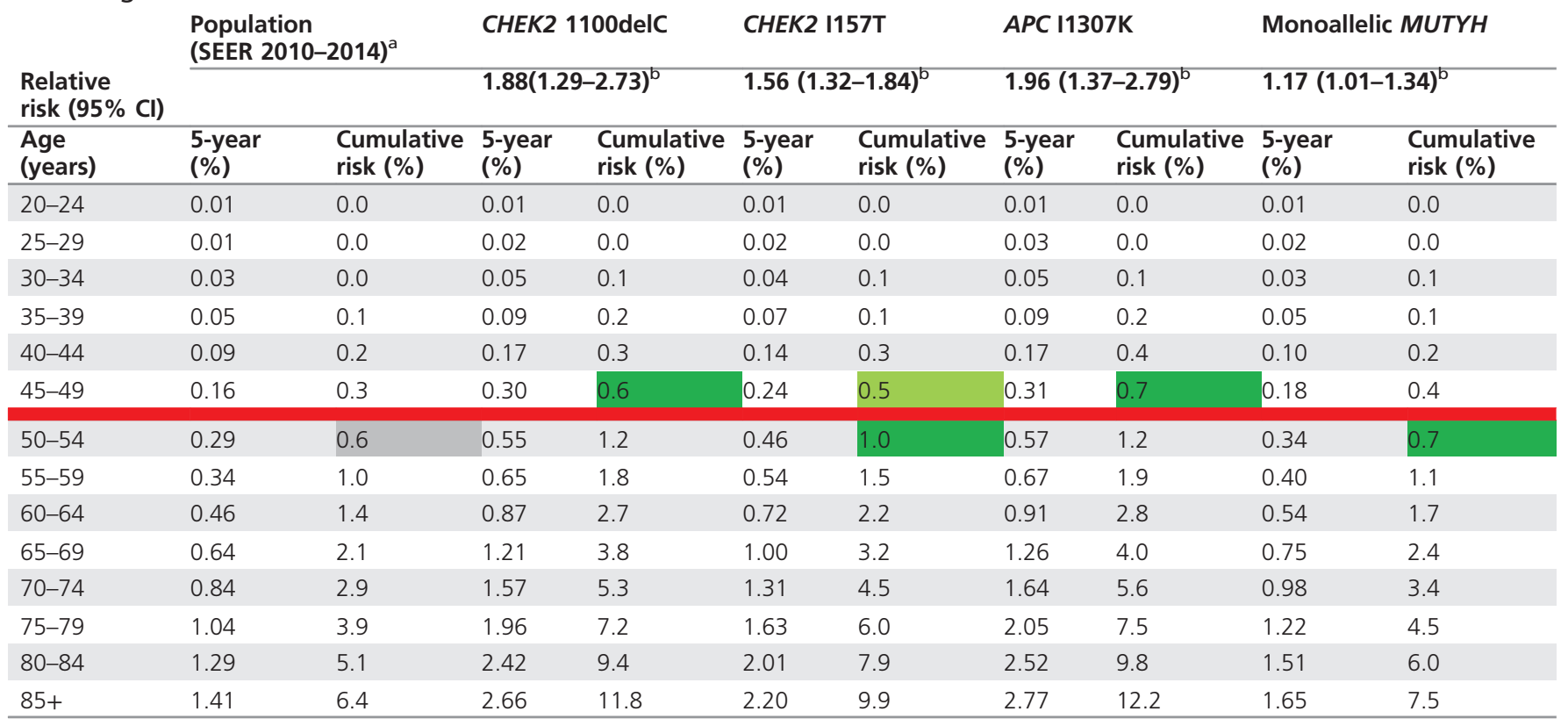

Box shading reflects the cumulative colorectal cancer risk level reached at colonoscopy initiation based on SEER data (gray box), and age ranges at which similar cumulative risk thresholds are reached (dark green box) or almost reached (light green box) according to gene mutation status.

$\mathrm{Cl}$, confidence interval; SEER, US Surveillance, Epidemiology, and End Results Program.

${ }^{a}$ Based on SEER cancer statistics, age-specific rates of cancer of the colon and rectum (invasive), 2010-2014 (ref. 4). ${ }^{b}$ Based on risk estimates in ref. 5. 
CRC have a 2.25 (95\% CI, 2.0-2.53) relative risk of CRC. ${ }^{11}$ As such, earlier and more frequent colonoscopy is recommended for individuals with a family history of CRC based on the strength of the family history and the age of the CRC diagnoses (Table 2). Importantly, when assessing the CRC risk in moderate-penetrance CRC gene carriers, the screening age and interval should be based on the family history when a family history-based assessment results in earlier and/or more frequent screening recommendations than those dictated by consideration of the moderate-penetrance gene variant alone.

While our analysis has several limitations including risk estimates derived from a single meta-analysis, estimates based on limited studies resulting in wide confidence intervals, and the assumption that gene-associated risk is constant over lifetime, these estimates encompass a multitude of studies and are currently the most representative of actual population risk. The use of SEER estimates for absolute-risk calculations may not be applicable to other countries with different populationspecific risks, or to populations such as African Americans, where absolute risk of CRC may be higher. Moreover, as SEER estimates are based on the inclusion of individuals at average risk as well as those at a higher risk of CRC, our derived risk estimates may reflect a slight overestimation of actual risk. Finally, our screening recommendations are based on the CRC CLTR threshold reached at initiation of screening colonoscopy at age 50 for the average-risk individual. Although it is conceivable that the age at which to initiate colonoscopy may be altered in the future, our risk-adapted screening strategy can be easily modified to meet such potential shifts in risk thresholds.

\section{CASCADE TESTING}

The utility of cascade testing is another important consideration with moderate-penetrance genes. As pathogenic CHEK2 variants confer an increased risk of breast cancer $^{7}$ with enhanced breast cancer screening already recommended, ${ }^{15}$ cascade testing in relatives of CHEK2 carriers is reasonable based on breast cancer risk alone, although it may not alter breast screening management in the presence of a strong breast cancer family history. Given the modest CRC risk associated with CHEK2 variants, and the resulting recommendation for more aggressive CRC screening, it also seems reasonable to offer cascade testing to relatives of CHEK2 carriers based on CRC risk alone to allow for individualized $\mathrm{CRC}$ risk management. For $A P C^{\star} \mathrm{I} 1307 \mathrm{~K}$ carriers, there are currently no additional cancer risk-reduction strategies that would be employed other than enhanced CRC screening. Given the modest, but significantly increased, risk of CRC associated with $A P C^{\star} I 1307 \mathrm{~K}$, as well as the resulting recommendations for earlier CRC screening for this population, an argument could also be made for offering cascade testing for this variant solely for CRC risk stratification. As CRC risk associated with monoallelic MUTYH mutations does not appear to merit increased CRC screening, cascade testing for this purpose alone may not be justified. However, given the high prevalence of monoallelic MUTYH carriers, cascade testing may be considered for family planning purposes, as recognition of carrier status in parents may help to identify offspring at risk for $M U T Y H$-associated polyposis due to biallelic MUTYH mutations. Finally, as future research may provide more clarity regarding CRC and possibly other cancer risks, the decision of whether to pursue cascade testing will likely continue to evolve.

\section{CONCLUSION}

Increased uptake of multigene panel testing will continue to lead to the detection of germ line variants that confer a modest increase in cancer risk with questionable associated clinical significance. In hereditary breast cancer, emerging literature ${ }^{16}$ suggests that many clinicians make inappropriately aggressive management recommendations for individuals with these variants. As CRC screening is not without its own risks, we advocate that a risk-adapted screening strategy be incorporated into the management of individuals with moderate-penetrance CRC susceptibility variants. For such germ line testing to truly translate into effective and rational risk reduction, larger studies will need to be performed, ideally with the inclusion of genetic and environmental modifiers (e.g., single-nucleotide polymorphisms, aspirin, etc.), to better quantify the risk associated with moderatepenetrance variants so that management recommendations can be more optimally tailored to the actual degree of CRC risk.

\section{ACKNOWLEDGMENTS}

Funding for this work was provided by National Institutes of Health/ National Institute of Diabetes and Digestive and Kidney Diseases grant 1K08DK106489 (B.W.K.), the Lustgarten Family Colon Cancer Research Fund (B.W.K.), Dana-Farber/Harvard Cancer Center SPORE in Gastrointestinal Cancer P50CA127003 (M.B.Y., J.E.G.), a Developmental Research Project Award (M.B. Y.), a Dana-Farber Cancer Institute Department of Medical Oncology Translational Research Grant (M.B.Y.), Memorial Sloan Kettering Cancer Center: National Cancer Institute Cancer Center Core Grant P30-CA008748 (K.O., M.E.R., Z.K.S.), and the Romeo Milio Lynch Syndrome Foundation (K.O., M.E.R., Z.K.S.).

\section{DISCLOSURE}

M.B.Y. receives research funding from Myriad. M.E.R. receives research funding from Myriad and Invitae. The other authors declare no conflict of interest.

\section{REFERENCES}

1. Tung N, Domchek SM, Stadler Z, et al. Counselling framework for moderate-penetrance cancer-susceptibility mutations. Nat Rev Clin Oncol 2016;13:581-588.

2. Yurgelun MB, Kulke MH, Fuchs CS, et al. Cancer susceptibility gene mutations in individuals with colorectal cancer. J Clin Oncol 2017;35: 1086-1095.

3. National Comprehensive Cancer Network. Genetic/Familial High-Risk Assessment: Colorectal (version 2.2017) and Colorectal Cancer Screening (version 2.2017). https://www.nccn.org/. Accessed 1 November 2017.

4. Howlader N, Noone AM, Krapcho M, et al. SEER Cancer Statistics Review, 1975-2014, National Cancer Institute. Bethesda, MD. https://seer.cancer. 
gov/csr/1975 2014/. Based on November 2016 SEER data submission posted to the SEER website April 2017.

5. Ma X, Zhang B \& Zheng W. Genetic variants associated with colorectal cancer risk: comprehensive research synopsis, meta-analysis, and epidemiological evidence. Gut 2014;63:326-336.

6. Song $\mathrm{H}$, Dicks $\mathrm{E}$, Ramus $\mathrm{SJ}$, et al. Contribution of germline mutations in the RAD51B, RAD51C, and RAD51D genes to ovarian cancer in the population. J Clin Oncol 2015;33:2901-2907.

7. Meijers-Heijboer $\mathrm{H}$, van den Ouweland A, Klijn J, et al. Low-penetrance susceptibility to breast cancer due to $\mathrm{CHEK} 2\left(^{*}\right) 1100$ delC in noncarriers of BRCA1 or BRCA2 mutations. Nat Genet 2002;31:55-59.

8. de Jong MM, Nolte IM, Te Meerman GJ, et al. Colorectal cancer and the CHEK2 1100delC mutation. Genes Chromosomes Cancer 2005;43:377-382.

9. Xiang HP, Geng XP, Ge WW \& Li H. Meta-analysis of CHEK2 1100delC variant and colorectal cancer susceptibility. Eur J Cancer 2011;47:2546-2551.

10. Katona BW \& Yang YX. Colorectal cancer risk associated with the CHEK2 1100delC variant. Eur J Cancer 2017;83:103-105.
11. Johns LE \& Houlston RS. A systematic review and meta-analysis of familial colorectal cancer risk. Am J Gastroenterol 2001;96: 2992-3003.

12. Liu C, Wang QS \& Wang YJ. The CHEK2 I157T variant and colorectal cancer susceptibility: a systematic review and meta-analysis. Asian Pac J Cancer Prev 2012;13:2051-2055.

13. Locker GY \& Lynch HT. Genetic factors and colorectal cancer in Ashkenazi Jews. Fam Cancer 2004;3:215-221.

14. Win AK, Dowty JG, Cleary SP, et al. Risk of colorectal cancer for carriers of mutations in MUTYH, with and without a family history of cancer. Gastroenterology 2014;146:1208-1211.

15. National Comprehensive Cancer Network. Genetic/Familial High-Risk Assessment: Breast and Ovarian (Version 2.2017). Available at: https:// www.nccn.org/. Accessed November 1, 2017.

16. Kurian AW, Li Y, Hamilton AS, et al. Gaps in incorporating germline genetic testing into treatment decision-making for early-stage breast cancer. J Clin Oncol 2017;35:2232-2239. 